\title{
The Creation of a Brain Atlas for Image Guided Neurosurgery Using Serial Histological Data
}

\author{
M. Mallar Chakravarty ${ }^{1}$, Gilles Bertrand ${ }^{1,3}$, Maxime Descouteaux ${ }^{2}$, \\ Abbas F. Sadikot ${ }^{1,3}$, and D. Louis Collins ${ }^{1}$ \\ 1 Montreal Neurological Institute (MNI), McGill University, Montreal Canada \\ 2 Center for Intelligent Machines (CIM), McGill University, Montreal Canada \\ 3 Division of Neurosurgery, McGill University, Montreal Canada
}

\begin{abstract}
Digital atlases of the human brain can help in the specific localization of structures of surgical relevance and interest in Image Guided Neurosurgery (IGNS). This paper outlines one of the steps in the creation of a digital atlas intended for IGNS, using histological data. The acquisition of histological data can include artefacts such as tearing, shearing, stretching, shrinking, as well as inhomogeneous staining leading to structural inhomogeneities. These inconsistencies are reduced using a non-linear intensity based registration procedure where deformations are defined using a maximized correlation coefficient estimate. The intensity artefacts brought about by inhomogeneous staining are reduced by applying a slice to slice intensity inhomogeneity correction by modelling the intensity mapping between slices as a third order polynomial that is estimated with a Least Trimmed Squared fit. The lateral ventricle was then segmented and to demonstrate increased smoothing along its surface.
\end{abstract}

\section{Introduction}

Though several anatomical imaging methods exist, these methods are not always adequate in planning neurosurgical procedures. Imaging modalities such as Magnetic Resonance Imaging (MRI), Positron Emission Tomography (PET), and Computed Tomography (CT) do not allow for a detailed analysis of certain structures in the brain due to their spatial resolution limitations. Digital or computerized atlases can help improve the accuracy and precision of the spatial localization of a region of interest within a patient's brain when used in conjunction with different imaging modalities 111.

The goal of Image Guided Neurosurgery (IGNS) is to use data from different imaging modalities in order to help plan stereotaxic neurosurgical procedures. Using this data, surgeons are able to interpret patient specific image volumes of anatomical, functional, and vascular relevance as well as their relationships. However, atlases can provide histological, functional or cyto-architectonic information to enhance a surgeon's visualization and understanding[111]. For example atlases of the basal ganglia and thalamus are required to determine stereotaxic targets for surgical treatment of movement disorders, such as tremor associated 
with Parkinson's disease [1. In the standard stereotaxic method, linear scaling is used to fit an atlas to a patient's anatomy [12. Our group was among the first to use a non-linear registration to improve atlas warping, thus enhancing positional targeting in patient's anatomy [11.

The creation of an atlas from histological data is a three-dimensional (3-D) problem and slices of histological data are two-dimensional (2-D). In most cases, anatomical structures are defined by an anatomist on the 2-D slices. This 2-D data must be reconstructed (tessellated) to create a 3-D geometric atlas that can be mapped to any patient's anatomy. Unfortunately, the acquisition of histological data contains artefacts such as tearing, shearing, stretching, shrinking or other types of morphological inconsistencies. If the 2-D data is reconstructed into 3 -D by simply stacking the $2-\mathrm{D}$ histological data, these morphological inconsistencies will be present in the reconstruction. This includes non-homogeneous structural definitions in addition to poorly defined and unsmooth surfaces. Inconsistent lighting and staining can also cause intensity inhomogeneities in reconstructions of the stacked histological data. In this paper we present initial work to correct the histological data by improving slice-to-slice alignment while correcting for some intensity artefacts.

Other work has been done in creating digital atlases using histological data. Ourselin et al. [8] used an intensity based block-matching strategy between slices of histological data and a Least-Trimmed Squared (LTS) minimization in order to define rigid or affine transformations from the source block to the target block. A 3-D registration from the reconstructed volume with a reference MRI was done using a similar block matching strategy. Toga et al.[13] used a fiducial marker based registration process to align serial sections. Their method uses a combination of local and differential scaling to put anatomical data in Talairach space [12]. Kim et al.[5] used a thin-plate spline (TPS)[2] technique to reconstruct a set of rat brain autoradiographs to a video block face reference. The TPS is a landmark based registration where the deformation between analogous landmarks is recovered using affine transformations. Kimet al. implemented their technique using landmarks defined on a grid or circle, that did not necessarily have any anatomical significance. Nowinski et al. 6] created a 3-D brain atlas database based on the atlases created by Talairach-Tournoux [12, Schaltenbrand and Wahren [10, and Ono, Kubik, Abernathey 7].

We propose a new technique in the creation of a digital atlas using histological data for which no complementary data (such as complementary MRI data or digital photographs of the blockface) is available. There are several cryogenic and histological datasets in existence which are of high quality, have been studied extensively, and pre-date the use of brain-imaging modalities or digital photography. The goal of our research is to create a 3-D reconstructed volume of such data while minimizing morphological variability introduced in the data acquisition process. Section 2 will describes the acquisition of the data while Section 3 describes the $3-\mathrm{D}$ reconstruction technique. 


\section{Histological Data Acquisition}

The histological data set used here was acquired in 1957 from a male patient who died of non-neurological complications. The brain was removed and fixed in $10 \%$ formalin and suspended in a gauze hammock to minimize any deformation. The brain was then split at the midline after which a block, centered on the thalamus and measuring $6 \mathrm{~cm}$ from front to back, $4.4 \mathrm{~cm}$ from top to bottom, and $3.2 \mathrm{~cm}$ from side to side, was sectioned out. The volume contains all of the basal ganglia together with the amygdala and the hippocampus (except for the posterior portion).

After dehydration the block was mounted in paraffin and placed in a microtome. Pairs of slices were taken at $0.70 \mathrm{~mm}$ intervals. Alternating sections were stained with Luxol Blue for myelin and with a Nissl stain for cell bodies. Corresponding myelin and cell photographs were then placed together and matched by hand using histological information. The contours were then segmented by hand by a neuroanatomical expert (GB) using Adobe Photoshop.

\section{3-D Reconstruction Methods}

The 3-D reconstruction used a two step approach. Prior to implementing the reconstruction scheme the contours and the histological images were separated. A picture of all three of these images can be seen in Figure 1. The colour photographs of the stained image were then converted to grey-level images. These images are considered in the reconstruction scheme described.

First a registration scheme was implemented in order to align homologous anatomical structures between sections. An intensity inhomogeneity correction was then implemented to correct for any lighting artefacts and inconsistent staining which may have occurred in the original data acquisition. These steps are described in the following sections.
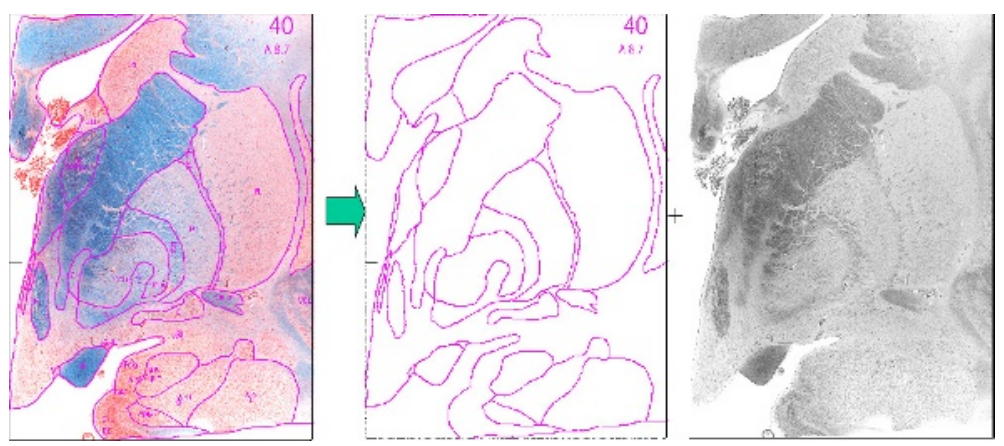

Fig. 1. An example of the data set. Left: The colour data with segmentation lines drawn. Middle: The segmentation contours. Right: Grey-level image. 


\subsection{Registration}

The Automatic Nonlinear Image Matching and Anatomical Labeling(ANIMAL) registration scheme was used to reduce the morphometric variability between slices [34]. The registration scheme is based on a 2-D lattice defined for each slice which makes up the volume. A deformation vector is then calculated for each node on the lattice. Each deformation vector is estimated by maximizing the correlation coefficient between source and target data of the local intensity neighborhood centered at each lattice point. Since we have no reference data, we maximize anatomical consistency between slices.

In doing the non-linear registration we consider the data sequentially in sets of three slices. Let the source be the second slice in the sequence of three slices. Let the two extreme slices in this set be the targets. All three slices in the set were blurred with an isotropic Gaussian kernel. Two different deformation vector fields were calculated to define the warp from the source slice to each of the target slices. The average of these two deformation vector fields was then applied to the source slice, thus maximizing its similarity with both targets simutaneously. We then incremented our procedure by a slice and consider the next set of three slice (the source slice from the previous step is now a target slice, and one of the target slices from the previous step is now a source slice). This procedure is done in a hierarchical fashion starting with blurred data to establish the initial fit. The blurring is reduced and the transforms are refined on the subsequent steps.

The voxel size of each slice was $34 \mu \mathrm{m} \times 34 \mu \mathrm{m} \times 700 \mu \mathrm{m}$. Based on this resolution, the blurring was done with a Gaussian kernel with a Full Width at Half Maximum (FWHM) of $640 \mu \mathrm{m}, 340 \mu \mathrm{m}$, and $204 \mu \mathrm{m}$. Deformation were calculated at each resolution in scale space and then applied to the sourced vector. The blurring was done to the results calculated at the previous step in scale space and then applied to the transformed images from that step. Parameter details for each step are given in Table 1 .

Registration parameters were verified using two arbitrary slices and calculating the deformation from the source to a target. The deformation was applied to the contours of the source and the chamfer distance between the transformed contours and the target was calculated. Using this technique a stiffness of 0.675 , a weight of 0.40 , and a similarity 0.05 were chosen for all resolutions in scale space.

Table 1. Parameters used in the Registration procedure for Different Resolutions in Scale Space.

\begin{tabular}{|c||c|c|c|}
\hline & Step 1 & Step 2 & Step 3 \\
\hline \hline FWHM $(\mu \mathrm{m})$ & 640 & 340 & 204 \\
\hline Step Size $(\mu \mathrm{m})$ & 1700 & 850 & 510 \\
\hline Lattice Diameter $(\mu \mathrm{m})$ & 3400 & 1700 & 1020 \\
\hline Sub Lattice & 30 & 20 & 20 \\
\hline
\end{tabular}


The algorithm for the registration procedure is given below.

1. for slice $=1$ to 84 set trasnformation[slice] to zero

2. for iter $=1$ to 3 blur all slices with blur[step]

- for slices $=1$ to 84

$--t_{1}=$ estimate of deformation[slice $i$ to slice $\left.i-1\right]$

$--t_{2}=$ estimate of deformation [slice $i$ to slice $\left.i+1\right]$

-- update transformation $[s]=\operatorname{average}\left(t_{1}+t_{2}\right)$

3. for slice $=1$ to 84 apply transformation $[s]$ to slice $s$

It should be noted here that this registration procedure will not account for any global deformations. Local anatomical inconsistencies between slices are accounted for by this process For example, uniform shrinkage throughout the data set cannot be accounted for since there is no reference. However this atlas is intended for use in an IGNS platform, therefore global scaling or non-linear transformation when registering the atlas to patient data should account for global deformations.

\subsection{Intensity Inhomogeneity Correction}

Intensity artefacts are also incurred during the acquisition of the histological data. Inhomogeneous lighting and inconsistent staining can cause artefacts in the intensity of the 3 -D reconstructed volume. The intensity inhomogeneity correction scheme developed by Prima et al. [9] was used. The technique models the intensity mapping of one image to another as a polynomial with degree greater than order one using an LTS fit. This mapping is then applied to all the pixels in the image.

Here we considered the data sequentially in groups of three slices. Much like in the registration case consider the middle slice the source and the two extreme slices the targets. The polynomials found through the LTS fit in this case were added. The procedure is then incremented by a slice, such that the new center slice is now the source slice. We consider the spatial variation between slices in the histological data set to be small enough to be accommodated by the technique. There is only one pass taken through the entire data.

\section{Results}

Figure 2 shows a pictoral view of the results after the registration step. The box represents the region of interest shown in Figure 3.

Figure 2 shows the spatially and intensity uncorrected and corrected data. Figures 3 shows a close of of both datasets, indicated by the box in Figure 2 . The close-up on the right shows that the contours have been smoothed.

To qualify the smoothness of the end result, the lateral ventricle was manually segmented at each slice in the volume. A surface rendering of this segmentation was done. Figure 4 below shows a view of the reconstructed ventricles of both the spatially corrected and uncorrected volumes. These surfaces can be seen in Figure 4. Smoothing has taken place along the edge of the ventricle demonstrated as shown in the close-up of the boxed areas. 


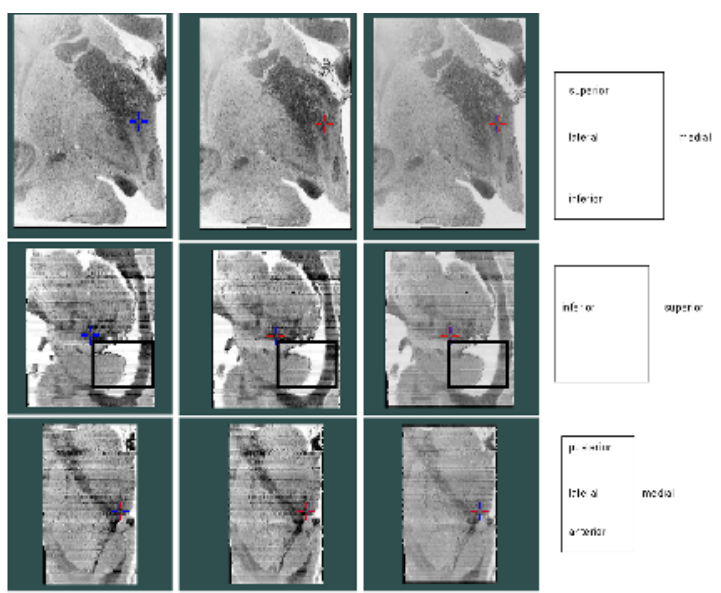

Fig. 2. Volumes before and after registration correction Left: The original, uncorrected data. Second from the Left: The post-registration output. Third from the Left The postregistration and intensity normalized output. Right: Orientation. Top: Coronal View. Middle: Saggital View. Bottom: Transverse View. The boxed region will be the focus of Figure 4.

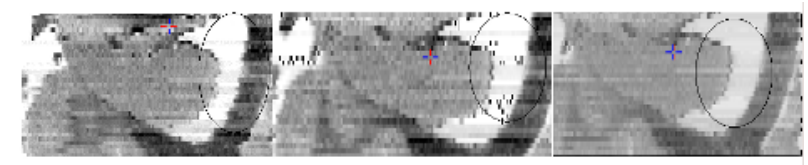

Fig. 3. Close-up of volumes before and after registration correction. Left: The data prior to all correction. Middle: Spatially corrected data. Right: Spacially and intensity corrected data. Smoothing of the edges have occurred. Minimization of streaking occurs in the intensity normalized volume/

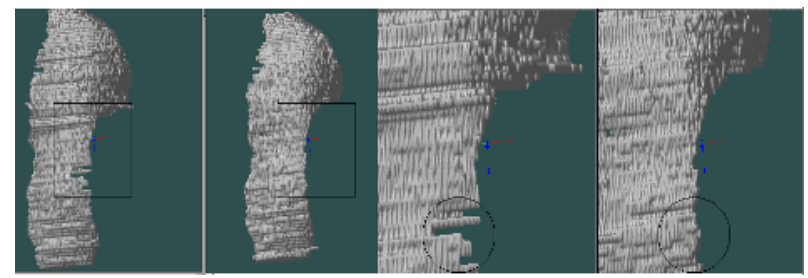

Fig. 4. First and Second Images from the Left: Uncorrected and corrected manually segmented ventricles. The box denotes the focus of the remainder of the image. Third and Fourth Images from the Left: Close ups of the boxes areas showing increased smoothing along the ventricle after spatial correction has been applied 


\section{Future Work and Conclusion}

This atlas is intended for use with an IGNS system and when complete, it would replace the simple stereotaxic atlas in use [11. This would be the first step towards a full integration of this atlas in an IGNS platform.

This work is also done with older data which has no block-face or MRI reference. This method should be tested against results obtained with reference data to see how it compares with such methods.

An intensity inhomogeneity scheme better suited to histological data is also necessary. Although the correction scheme used here yields global slice-to-slice intensity correction, it is not optimized for spatially variant inhomogeneity between slices. An adaptive thresholding technique, is being considered. This technique would examine a neighborhood of pixels and use a polynomial fit between a reference slice and the target slice. When the registration and intensity normalizations steps have been completed, we will continue to the next phase of our work where the contours will be deformed using the same transformations defined in the registration steps mentioned above. Also, 3-D geometrical and anatomical atlases need to be generated in order to integrate this atlas into an IGNS system.

We have presented here a method to reconstruct $3 \mathrm{D}$ volume from $2 \mathrm{D}$ histological data, while accounting for anatomical inconsistencies and slice to slice intensity inhomogeneities. The results demonstrate increased smoothness of the reconstructed volume. This will allow us to proceed in creating a 3-D geometric atlas of the basal ganglia and thalamus for use in stereotaxic IGNS.

\section{References}

[1] J. Atkinson, D.L. Collins, G. Bertrand, T.M. Peters, B. Pike, and A.F. Sadikot. Optimal location of thalamotomy lesions for tremor associated with Parkinson disease: a probablisitic analysis based on postoperative magnetic resonance imaging and integrated digital atlas. Journal of Neurosurgery, 96(5): 672-680, October 2002 .

[2] F.L. Bookstein. Principal Warps: Thin-Plate Splines and the Decompostion of Deformations. IEEE Transactions on Pattern Analysis and Machine Intelligence, 11(6): 567-585, 1989.

[3] D.L. Collins, P. Neelin, T.M. Peters, and A.C. Evans. Automatic 3D Intersubject Registration of MR Volumetric Data in Standardized Talairach Space. J. of Computer Assisted Tomography, 18(2): 192-205, March 1994.

[4] L. Collins, T.M. Peters, and A.C. Evans. An Automated 3D non-linear image deformation procedure for Determination of Gross Morphometric Variability in Human Brain. In Richard A. Robb, editor, VBC, volume 2359 of SPIE, Rochester (Minnesota) (USA), 1994.

[5] B. Kim, J.L. Boes, K.A. Frey, and C.R. Meyer. Mutual Information for Automated Unwarping of Rat Brain Autoradiographs. Neuroimage, 5: 31-41, 1997.

[6] W.L. Nowinski, A. Fang, B.T. Nguyen, J.K. Raphel, L. Jagannathan, and R. Raghavan. Multiple Brain Atlas Database and Atlas Based Neuroimaging System. Computer Aided Surgery, 2: 42-66, 1997. 
[7] M. Ono, S. Kubik, and C.D. Abernathy. Atlas of the Cerebral Sulcii. Georg Thieme Verlag/Thieme Medical Publishers, Stuttgart, Germany, 1990.

[8] S. Ourselin, E. Bardinet, D. Dormont, G. Malandain, A. Roche, N. Ayache, D. Tandé, K. Parain, and J. Yelnik. Fusion of histological sections and mr images: Towards the construction of an atlas of the human basal ganglia. In Medical Image Computing and Computer-Assisted Intervention (MICCAI), pages 743-751, Utrecht, Netherlands, October 2001.

[9] S. Prima, N. Ayache, A. Janke, S.J. Francis, D.L. Arnold, and D.L. Collins. Statisitical Analysis of longitudinal MRI data: applications of disease activity in MS. In Fifth International Conference on Medical Image Computing and Computer-Assisted Intervention, MICCAI'2002, Lecture Notes in Computer Science, Tokyo, Japan, 2002. Springer.

[10] G. Schaltenbrand and W. Wahren. Atlas for Stereotaxy of the Human Brain. Georg Thieme Verlag, Stuttgart, Germany, 1977.

[11] P. St-Jean, A.F. Sadikot, L. Collins, D. Clonda, R. Kasrai, and A.C. Evans. Automated Atlas Integration and Interactive Three-Dimensional Visualization Tools for Planning and Guidance in Functional Neurosurgery. IEEE Transactions on Medical Imaging (TMI), 17: 854-866, May 1998.

[12] J. Talairach and P. Tourneau. Co-Planar Stereotaxic Atlas of the Human Brain. Georg Thieme Verlag, Stuttgart, Germany, 1988.

[13] A.W. Toga, A. Goldkorn, K. Ambach, K. Chao, B.C. Quinn, and Y. Yao. Postmortem Cryosectioning as an Anatomic Reference for Human Brain Mapping. Computerized Medical Imaging and Graphics, 21(2): 131-141, 1997. 